\title{
Finite Coherence Length of Thermal Noise in Percolating Systems
}

\author{
K. K. Bardhan and C. D. Mukherjee \\ Saha Institute of Nuclear Physics, 1/AF Bidhannagar, Calcutta 700 064, India
}

(Dated: November 2, 2018)

\begin{abstract}
Noise has been measured in two types of coductor-insulator mixtures as a function of bias and composition. It was marked by a huge increase in magnitude as the resistance increased only slightly due to Joule heating. The noise (resistance) current scale $I_{s}\left(I_{r}\right)$ for nonlinearity were found to scale with the linear resistance $R_{o}$ as $I_{s}\left(I_{r}\right) \sim R_{o}{ }^{-x_{s}\left(x_{r}\right)}$ where the exponent $x_{s}$ is equal to 0.80 and 0.68 in carbon-wax and carbon-polyethylene respectively and $x_{r} \approx 0.5$. It is shown that the large increase of noise in nonohmic regime as well as the differences between the noise and resistance exponents are due to the finite-sized inequilibrium thermal fluctuations whose coherence length is same as the correlation length of the underlying percolating systems. A expression for $x_{s}$ is derived.
\end{abstract}

PACS numbers: $72.70 .+\mathrm{m}, 72.20 . \mathrm{Ht}, 72.60 .+\mathrm{g}$

The low frequency resistance noise, also known as $1 / f$ noise, is a very common phenomenon [1] in normal conductors and is increasingly being used as a tool for studying, particularly, disordered systems [2]. While its origin remains controversial, some of its features are wellestablished. In particular, the correlation length of resistance fluctuations $\left(\xi_{s}\right)$ is commonly assumed to be of the order of microscopic lengths 1]. This implies that the noise power should be inversely proportional to the number of fluctuators or the system volume $L^{d}, d$ being the system dimensionality. But there are cases where relatively large increases in the noise levels under certain experimental conditions have been explained in terms of coarsening of the coherent volume with correlation lengths increasing to macroscopic scale. This, other things remaining same, amounts to an increase in the noise level by a factor of $\left[L /\left(L / \xi_{s}\right)\right]^{d}=\xi_{s}^{d}$ with $\xi_{s} \leq L$. For example, the large increase in the noise at the onset of charge density wave motion in certain conductors upon application of an electric field has been ascribed to the finite coherence length of a charge density wave domain 3 . Analogous phenomena are the well-know critical opalescence in various systems [4] where the static correlation length diverges as a critical point is approached. Interestingly, in systems in nonequilibrium states but not necessarily near any critical point, the correlation length of fluctuations at equal time is also of macroscopic order $[5]$.

Earlier [ $[$ ], the resistance behaviour of various composite samples (random mixtures of conductors and insulators) exhibiting substantial Joule heating was invesigated as a function of biasing currents. At low currents, the resistance $R=V / I$ had a bias-independent value $R_{o}$ but increased at high currents. It was found that the current $I_{r}$ at which the sample resistance starts increasing scaled with $R_{o}$ as $I_{r} \sim R_{o}^{-x_{r}}$ where $x_{r} \approx 0.5$ is the onset exponent for resistance. In this letter, we report on systematic measurements of low frequency resistance noise as a function of currents in two composites systems of carbonwax (C-W) and carbon-polymer (C-HDPE). Samples under fixed currents were in nonequilibrium steady states (below breakdown) with temperature gradients. It was found that (i) the relative noise $\mathcal{S}$ (see below) behaved in a similar fashion as the resistance such that the current scale $I_{s}$ for onset of nonlinearity in noise 7] and the associated onset exponent $x_{s}$ could be defined as before: $I_{s} \sim R_{o}^{-x_{s}} . x_{s}$ was found to be larger than $x_{r}$ :

$$
x_{s}=\left\{\begin{array}{l}
0.80 \pm 0.03(\mathrm{C}-\mathrm{W}) \\
0.68 \pm 0.04(\mathrm{C}-\mathrm{HDPE})
\end{array}\right.
$$

(ii) The noise $\mathcal{S}$ of a sample as a function of resistance increased tremendously compared to that caused by varying static disorder (i.e. composition). It is argued that such increase in noise reslults from additional resistance fluctuations induced by thermal fluctuations which are coherent over a length of the correlation length $\xi$ of the underlying percolation structure i.e. $\xi_{s} \approx \xi$. It is shown that this assumption leads to an expression for $x_{s}$ :

$$
x_{s}=x_{r}+d \nu / 4 t-w_{J} / 4
$$

where $\nu$ and $t$ are percolation correlation and conductivity exponents respectively $[8]$ and $w_{J}$ is the noise exponent [6]. Using $d=3, \nu=0.9[8$ and experimental values of $x_{r}, t$ and $w_{J}$ in (2) yields $x_{s}=0.83 \pm 0.10$ and $0.67 \pm 0.05$ for $\mathrm{C}-\mathrm{W}$ and $\mathrm{C}-\mathrm{HDPE}$ respectively. These values agree well with the experimental ones in (11).

Noise have been studied extensively in many percolating systems like composites both experimentally and theoretically but mostly in ohmic regimes $\underline{\underline{9}}$. Recently, the bias-dependent behaviour of noise near the percolation threshold has been measured [10]. A composite sample is primarily characterised by its conductor fraction $p$. Above a particular value $p_{c}$, called percolation threshold, a continuous path is formed from one end of the sample to another, enabling current to flow through the system. A sample possesses a natural length scale which is the two-particle correlation length $\xi$ given by $\xi \sim\left(p-p_{c}\right)^{-\nu}$. The noise (in the ohmic regime) tends to diverge 11] as the conducting fraction $(p)$ is reduced from large values above $p_{c}$. However, this divergence 
has been explained to originate entirely from the static geometrical disorder 12 . $\xi_{s}$ remains of the order of microscopic scales and independent of $\xi(p)$. Electrical conductivity of a composite sample is characterised by the exponent $t$, defined by $R_{o} \sim\left(p-p_{c}\right)^{-t}[\underline{8}]$. The resistance $R_{o}$ decreases with increasing $p$. The noise exponent $w_{J}$ is defined by $\mathcal{S}(I \sim 0) \sim R_{o}^{w_{J}}$.

The carbon-wax system is the same one as used earlier for noise measurements in the range $p \geq p_{c}[10]$ but samples used in this work were in the Joule range $p>p_{J}$. The fraction $p_{J}>p_{c}$ is such that the resistance of a sample in response of an applied field increases if $p>p_{J}$. That the increase in resistance in this range of $p$ is indeed due to the Joule heating has been confirmed by a number of observations 13. like the sensitivity of measured values of resistances to the presence of a cooling fan near samples. For this system, $p_{c}=0.76 \%$ by volume, $p_{J}<3 \%$ and $t=2.05 \pm 0.1514$. C-W samples were all disk-shaped, $10 \mathrm{~mm}$ in diameter and $6 \mathrm{~mm}$ high. Connections to electrodes were such that current flowed parallel to the thickness of the disc. The preparation and characterisation of C-HDPE samples have been described in ref. [15]. $t$ has the value of $2.9 \pm 0.1$ in this system. These samples had dimensions $10 \times 4 \times 1 \mathrm{~mm}^{3}$. In this case, currents flowed parallel to the longest side. All measurements were done at room temperature at constant currents. For any constant current, sufficient time (more than $40 \mathrm{~min}$ ) was allowed for the sample resistance to attain a steady value. Furthur details of noise measurements can be found in ref. 10.

Let $S_{V}$ denote the spectral power of voltage fluctuations at a constant current. Fig. 1 shows plots of $S_{V}(0.5 H z)$ vs. $I$ for five C-W samples with $p$ as indicated. At low currents (ohmic regime), $S_{V}$ varied as $I^{2}$ (solid straight lines). At high currents, $S_{V}$ varied faster than $I^{2}$ in the similar manner as $V-I$ curves become nonlinear. Such concurrent nonlinear behaviour of noise [7] and resistance becomes more evident in Fig. 2 where the normalised relative noise $\mathcal{S}$, defined by $\mathcal{S}=S_{V} / V^{2}$, (squares) and conductance $\sigma=1 / R$ (circles) are shown as functions of the current for the two C-W samples with $p=3.5$ (closed symbols) and 10\% (open symbols). The conductance rather than resistance was used to avoid overlapping of data. The relative noise and the resistance of a sample have similar qualitative behaviour in that both remain close to their respective zero-bias values at small currents but appear to deviate from those values as the latter is increased. The currents, $I_{s}$ and $I_{r}$, at which onset of nonlinearity took place were determined by two independent methods. In the first method, they were determined by adopting the criteria that corresponded to the increase in noise by $100 \%$ and the decrease in conductance by $5 \%$. These levels of changes are indicated by the dotted lines in Fig. 2. The criterion of $100 \%$ change of noise level was adopted to increase the accuracy of the determination of $I_{s}$ as the noise increased

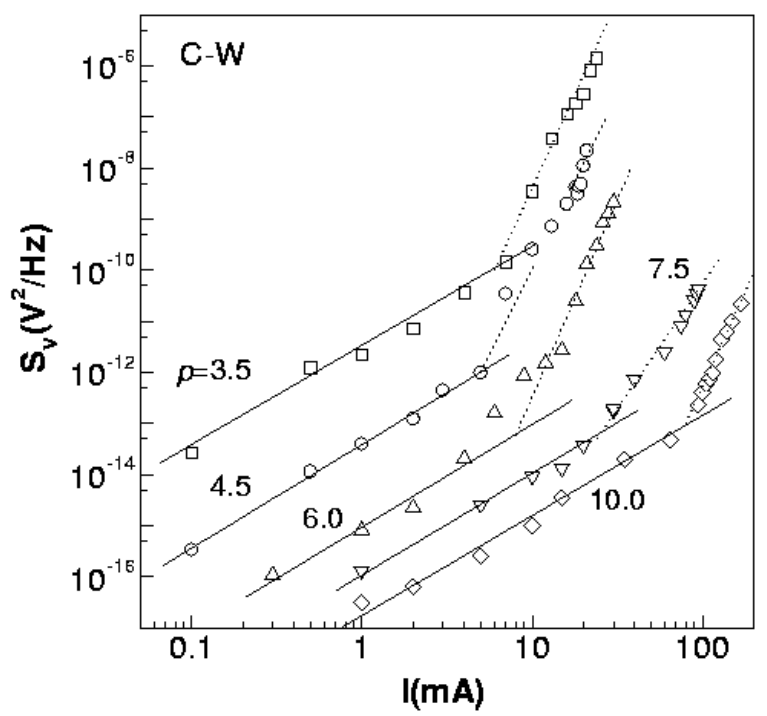

FIG. 1: Plots of noise power $S_{V}(0.5 H z)$ vs. dc current $I$ in five carbon-wax samples with carbon percentage by volume $(p)$ as marked. The solid lines are fits to a power law with all slopes equal to 2 and the dashed curves are only guide to the eye. For clarity, the data corresponding to $p=3.5$ and $4.5 \%$ have been shifted upward by factors of 100 and 10 respectively.

steeply by several orders of magnitude while the conductance decreased only by an order of unity. As $p\left(R_{o}\right)$ increases (decreases), both $I_{s}$ and $I_{r}$ increase. Log-log plots of both $I_{r}$ (circles) and $I_{s}$ (squares) vs. $R_{o}$ in the two systems are shown in Fig. 3. Straight lines indicate power-law fits with the slopes as indicated. In the second method, the currents for each sample was scaled with respect to $I_{s}$ determined by trial and error such that the curves $\mathcal{S}(I) / \mathcal{S}(0)$ vs. $I / I_{s}$ of all samples of each system collapsed on a single curve 13]. Similar procedures were followed with resistance data for $I_{r}$ (see Fig. 3 of ref. 6 ). This method yielded $x_{r}=0.53 \pm 0.02$ and $0.45 \pm 0.02$ and $x_{s}=0.81 \pm 0.03$ and $0.68 \pm 0.04$ in $\mathrm{C}-\mathrm{W}$ and C-HDPE respectively. Averages of $x_{s}$ from the two methods are shown in (11) and averages of $x_{r}$ were used in (2). $w_{J}$ was obtained from the relative noise in ohmic regimes, $\mathcal{S}_{0}$. Its value in C-HDPE was found to be $0.13 \pm 0.06[\underline{6}]$. But, the data of $\mathrm{C}-\mathrm{W}$ had large scatter. As a consequence, a power-law fit yielded $w_{J}=0.11 \pm 0.21[13$. These values in Joule samples, significantly smaller than those near $p_{c}$, were used in (2) to obtain finally the predicted values as mentioned above.

The most significant feature of the nonlinear noise in the Joule regime is, of course, the huge increase in magnitude compared to the small change in sample resistance. This is furthur emphasised by plotting (curve b) the relative noise in a Joule sample (C-W, $p=10 \%)$ against the bias-dependent resistance $R$ in Fig. 4 which also shows the variation (curve a) of relative noise in $\mathrm{C}-\mathrm{W}$ with static disorder characterised by the linear resistance 


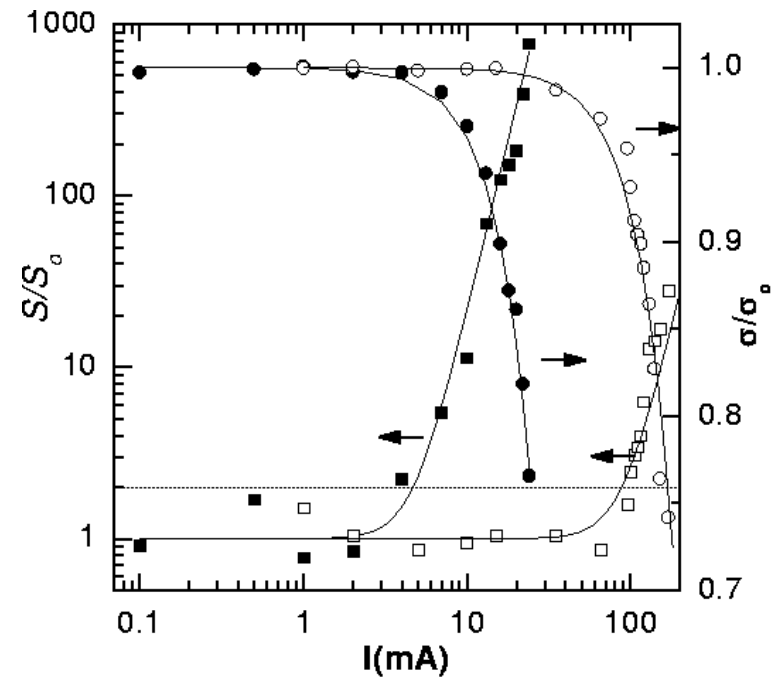

FIG. 2: Normalised relative noise power (squares) and conductance (circles) are shown as functions of $I$ for two C-W samples with $p=3.5 \%$ (closed symbols) and $10.0 \%$ (open symbols). The solid lines are fits to the functionals $\mathcal{S} / \mathcal{S}(0)=$ $1+a_{s} I^{4}$ and $\sigma / \sigma_{o}=1-a_{r} I^{2}$ where $a_{s}\left(a_{r}\right)=2.1 \times 10^{9}(400)$ and $1.7 \times 10^{4} \mathrm{~A}^{-4}\left(8.5 \mathrm{~A}^{-2}\right)$ for $p=3.5$ and $10 \%$ respectively. The dashed lines indicate the criteria for determining the current scales for onset of nonlinearity (see text).

$R \equiv R_{o}(p)$. Let's note that all measurements in this work were carried out in the steady and reversible states below breakdown [6] and hence, no change in the topology of percolating networks is expected. It is then clear that small changes in resistances of the network elements alone can not account for the large increase of the noise level. Indeed we show below that temperature fluctuations which modulate resistances are responsible for this phenomenon. The local rise in temperature results in a change in the local resistance $r$ determined by the temperature coefficient of resistivity, $\beta=(1 / r) d r / d T$. The final state under a constant current condition and a positive $\beta$ is determined by the balance between the heat generated and the loss of heat to the environment per unit time.

The electrical response to Joule heating in percolating networks has been treated in detail by several authors 6 , 16, 17. In such a network, the changed resistance due to Joule heating, in the first approximation, is given by 17 .

$$
R=R_{o}+a \beta h R_{o}^{2} \mathcal{S}_{o} I^{2}
$$

Here $a$ is a simple constant. $h$ is the heat transfer coefficient, defined as the ratio between temperature rise and power generated in a conducting element. $\mathcal{S}_{o}=\mathcal{S}(0)$ is the relative noise power in the ohmic regime (Figs. 1 and 2). Each solid line through conductance data in Fig. 2 is a fit to the expression $1-a_{r} I^{2}$ according to Eq. 3. Here, $a_{r}=a \beta h R_{o} \mathcal{S}_{o}$. The current scale $I_{r}$ for the onset of nonlinearity in resistance is given by $I_{r} \sim\left(a \beta h R_{o} \mathcal{S}_{o}\right)^{-1 / 2}=a_{r}{ }^{-1 / 2}[\underline{6}$ ]. It may be noted that

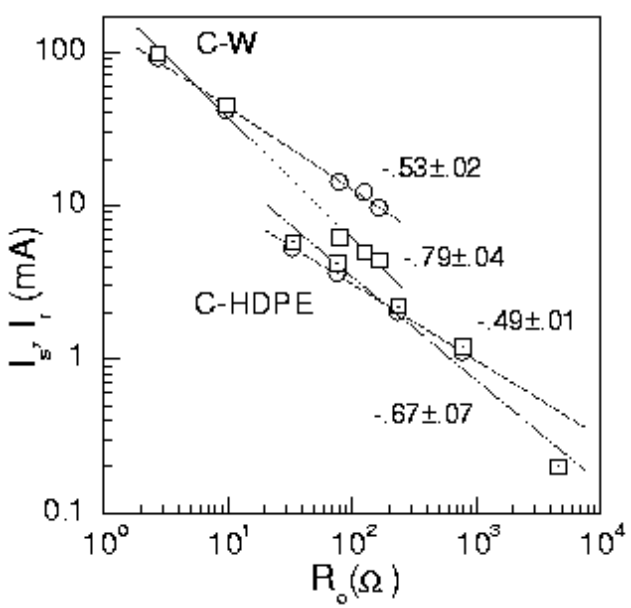

FIG. 3: Plots of nonlinearity current scales $I_{s}$ (noise, squares) and $I_{r}$ (conductance, circles) vs. zero-field resistance $R_{o}$ for two systems. Data of C-HDPE samples have been shifted down by a factor of 5 . The solid lines are the power law fits to the data with the exponents as indicated.

the quantity $h$, as defined above, strives to provide, in the spirit of mean-field approach, a reasonable description of heat conduction in inhomogeneous media, which otherwise poses a formidable problem. Ultimately, it governs the process of exchange of heat between the sample and the environment [16] and hence, is quite susceptible to fluctuations.

Eq. 3 formed the basis for obtaining the linear noise by the so called third harmonic measurements 16. It will now be used to calculate the noise in the nonlinear regime. A small variation $\delta R$ is given by $\delta R / R=$ $\left(1+a_{r} I^{2}-2 a_{r}^{2} I^{4}\right) \delta R_{o} / R_{o}+a_{r} I^{2}\left(1-a_{r} I^{2}\right) \delta h / h$ after neglecting variation of $\mathcal{S}_{o}$. Using this and assuming that fluctuations in the ohmic regime $R_{o}$ are uncorrelated with those in $h$ (or thermal fluctuations), i.e. $\left\langle\delta h \delta R_{o}\right\rangle=0$, it could be easily verified that $\mathcal{S}=<\delta R^{2}>/ R^{2}$ is given by

$$
\mathcal{S} / \mathcal{S}_{o} \simeq 1+2 a_{r} I^{2}+\left(<\delta h^{2}>/ h^{2} \mathcal{S}_{o}-3\right) a_{r}{ }^{2} I^{4}
$$

after keeping terms up to the order of $I^{4}$. According to (3), $a_{r} I^{2}$ is equal to the fractional change of a sample resistance at current $I$ which is of the order of 1 (see refs. 6 and 18 for its maximum possible values). Since the relative noise, in contrast, increases in the same range of currents by several orders of magnitude (see Fig. 2) we must have $\left\langle\delta h^{2}>/ h^{2} \gg \mathcal{S}_{o}\right.$ so that Eq. (4) reduces to

$$
\mathcal{S} / \mathcal{S}_{o} \simeq 1+\left(<\delta h^{2}>/ h^{2} \mathcal{S}_{o}\right) a_{r}{ }^{2} I^{4}
$$

Fits to the noise data according to (5) with $a_{s}=$ $\left(<\delta h^{2}>/ h^{2} \mathcal{S}_{o}\right) a_{r}{ }^{2}$ are shown in Fig. 2. The values of $a_{s}$ and $a_{r}$ given in Fig. 2 yield $<\delta h^{2}>/ h^{2} \mathcal{S}_{o} \sim 10^{4}$ to $10^{2}$ in the range of $3.5-10 \%$ of $p$, much greater than 1. Goodness of fittings proves the earlier assertion that it is the secondary (i.e. thermal) source of noise that is responsible 


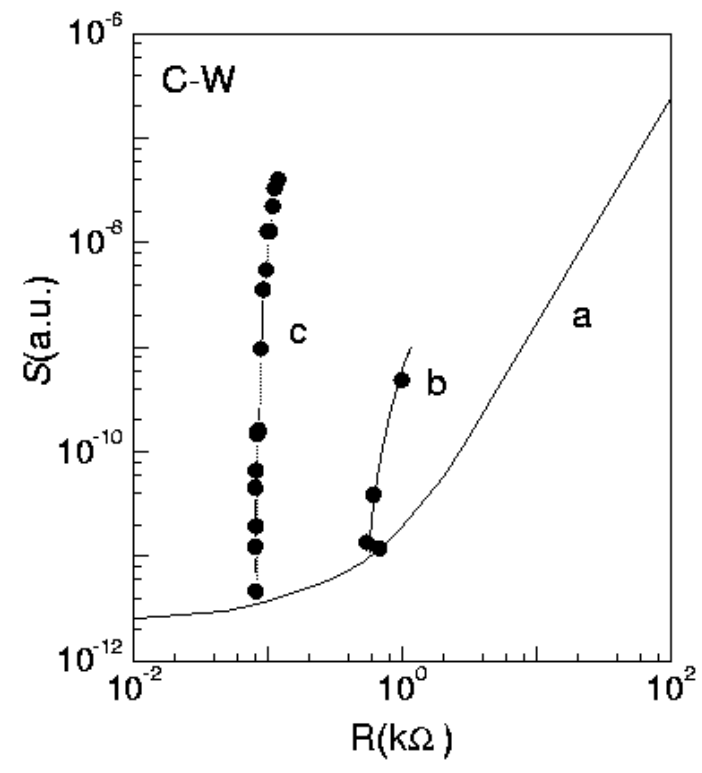

FIG. 4: Comparision of noise in nonohmic regime with that in ohmic regime of a composite system. Relative noise is plotted against $R$ for two cases: for curve (b), $R$ is the bias-dependent resistance of a joule sample $(p=10 \%)$ and for curve (a), $R$ is the composition-dependent zero-bias resistance. High resistance data of curve (a) are taken from ref. 10.

for a huge increase in noise level in the nonlinear regimes in Joule samples. The primary source is, of course, the one responsible for $\mathcal{S}_{o}$. Let us now consider the possible origin of such large magnitude of the thermal noise.

The inhomogeneous nature of percolating networks give rise to randomly occuring 'hot spots' which are the regions carrying high current densities and get heated most. Consequently, thermal gradients result in steady heat flows away from those spots, thus setting up nonequilibrium steady states. Now, a percolating system according to the 'Node-Link-Blob' picture 8] could be viewed as a homogeneous system on the scale of the correlation length $\xi(p)$. Links i.e. singly connected bonds in this picture are the hot spots. With such multiple sources within the length $\xi(p)$ and the fact that the fluctuation correlation length at equal times in nonequilibrium states increases to macroscopic size [5] it is assumed that a volume of $\xi^{d}$ will fluctuate thermally in a coherent manner i.e. thermal $\xi_{s}$ will simply snap onto $\xi$. This means that $<\delta h^{2}>/ h^{2} \sim \xi^{d}$. Such coarsening of coherent length in inhomogeneous systems explains the large increase in the thermal noise. It is quite instructive to compare thermal fluctuation $\left\langle\delta T^{2}>/ T^{2}\right.$ in the Joule regime with that in equilibrium. The latter at room temperature is roughly inversely proportional to the number of particles in a sample i.e. $10^{-20}$ whereas the former is approximately equal to $<\delta h^{2}>/ h^{2} \sim 10^{-8}$ to $10^{-10}$ with $\mathcal{S}_{o} \approx 10^{-12}$ at $0.5 \mathrm{~Hz}$. Note that in a homogeneous sample under a thermal gradient, $\xi_{s}$ can still be of micro- scopic size 1]. From (15), the current scale for nonlinear noise is given by $I_{s}^{-1} \sim\left(<\delta h^{2}>/ h^{2} \mathcal{S}_{o}\right)^{1 / 4} a_{r}{ }^{1 / 2}$ which, upon using $\xi \sim R_{o}^{\nu / t}$ and $a_{r}=I_{r}^{-2}$, yields the relation (2). Note that the latter predicts $x_{s}-x_{r}$ to have theoretical values of 0.34 and 0.5 in $3 \mathrm{D}$ and $2 \mathrm{D}$ respectively ignoring $w_{J}$ and hence, the exponents to be more divergent in two dimension.

In conclusion, we presented an experimental study involving two distinct noise sources with different coherence lengths. In the present case, the secondary thermal noise was induced by the Joule heating and became dominant in the nonohmic regime due to amplification by coarsening of the coherence length. This points to possible furthur use of noise study as a tool in systems such as manganites 19. which exhibit multiple phases having very likely different fluctuation properties.

We are grateful to M. B. Heaney for the carbonpolyethylene samples. We acknowledge the assistance of Arindam Chakrabarti in the preparation of $\mathrm{C}-\mathrm{W}$ samples.

[1] S. Kogan, Electronic noise and fluctuations in solids (Cambridge University Press, Cambridge, 1996).

[2] M. B. Weissman, Adv. Rev. Mater. Sci. 26, 395 (1996).

[3] S. Bhattacharya et al., Phys. Rev. Lett. 54, 2453 (1985).

[4] A. Münster, in Fluctuation phenomena in solids, Ed. R. E. Burgess (Academic Press, New York, 1965).

[5] R. Schmitz, Phys. Rep. 171, 1 (1988).

[6] C. D. Mukherjee, K. K. Bardhan, and M. B. Heaney, Phys. Rev. Lett 83, 1215 (1999).

[7] Some authors (e.g. C. Perman et al., Phys. Rev. Lett. 67, 2529 (1991)) termed the noise as nonlinear when it did not vary as the square of the applied bias in the ohmic regime. The term 'nonlinear noise' is used here to describe, in analogy with nonlinear conductance, situations where the relative noise power shows dependence on the applied bias.

[8] D. Stauffer and A. Aharony, Introduction to percolation theory (Taylor and Francis, 2nd. ed, London, 1992).

[9] For a brief review see A.-M.S. Tremblay, B. Fourcade, and P. Breton, Physica A 157, 89 (1989).

[10] U. N. Nandi, C. D. Mukherjee, and K. K. Bardhan, Phys. Rev. B 54, 12903 (1996).

[11] C. C. Chen and Y. C. Chou, Phys. Rev. Lett. 54, 2529 (1985).

[12] R. Rammal et al., Phys. Rev. Lett. 54, 1718 (1985).

[13] Details to be published elsewhere.

[14] R. K. Chakrabarty, K. K. Bardhan, and A. Basu, Phys. Rev. B 44, 6773 (1991).

[15] M. B. Heaney, Phys. Rev. B 52, 12477 (1995).

[16] Y. Yagil and G. Deutcher, Phys. Rev. B 46, 16115 (1992).

[17] M. A. Dubson et al., Phys. Rev. B 39, 6807 (1989).

[18] U. N. Nandi, C. D. Mukherjee, and K. K. Bardhan, Physica B 72, 279 (2000).

[19] Y. Tokura, Colossal-Magnetoresistive Oxides, Ed. Y. Tokura (Gordon \& Breach Sci. Pub., New York, 1999). 